Check for updates

Cite this: RSC Adv., 2019, 9, 8310

Received 9th January 2019

Accepted 26th February 2019

DOI: 10.1039/c9ra00195f

rsc.li/rsc-advances

\section{A novel and facile synthesis of visible photoluminescence Si nanocrystals by room temperature mechanochemical disproportionation of $\mathrm{SiO}$}

\author{
Yunzi Xin, (D) Yuping Xu, Jeongbin Lee and Takashi Shirai*
}

Visible photoluminescence $\mathrm{Si}$ nanocrystals ( $\mathrm{Si} \mathrm{NCs}$ ) are synthesized via a novel and facile room temperature mechanochemical disproportionation of SiO. The size of the synthesized Si NCs can be easily tuned by the mechanochemical conditions. Surface passivated $\mathrm{Si}$ NCs exhibit excitation wavelength-dependent photoluminescence, which is attributed to quantum confinement effects.

Si nanocrystals (NCs) have attracted much attention as promising materials for optoelectronic devices, photovoltaics, and in vivo bio-imaging based on their broadly size-tunable bandgap due to quantum confinement effects, natural abundance, non-toxicity and good biodegradability. ${ }^{1-16}$ As for optoelectronic devices, Maier-Flaig et al. ${ }^{3}$ and Xin et al. ${ }^{4}$ developed Si NC light-emitting diodes giving electroluminescence in red and white-blue wavelengths, respectively, whose research provides a new opportunity for next-generation solid-state lighting and large-area display technology. Tan and $\mathrm{Ni}$ et $a .^{5}$ developed broadband (from the ultraviolet to near-infrared region) optoelectronic synaptic devices by utilizing Si NCs that can help advance neuromorphic computing. In the case of photovoltaic applications, Kim et al. reported highly efficient Si NC heterojunction solar cells recently. ${ }^{6}$ Meinardi et al. reported Si NC based highly efficient luminescent solar concentrators, and their flexible device with comparable performance to flat concentrators provides new design freedom for building integrated photovoltaic elements. ${ }^{7}$ By applying boron-doped Si NCs, $\mathrm{Ni}$ and $\mathrm{Ma}$ et al. fabricated graphene-based hybrid phototransistors and obtained remarkable high sensitivity ultrabroadband (from ultraviolet to mid-infrared region) photodetection by taking advantage of the localized surface plasmon resonance of boron-doped $\mathrm{Si} \mathrm{NCs}^{8}$ Numerous syntheses of photoluminescent Si NCs have been reported, which can be divided into top-down and bottom-up approaches. In the case of top-down approaches, laser ablation of Si wafer, ${ }^{9}$ electrochemical etching of $\mathrm{Si}^{10}$ and mechanochemical ball-

Advanced Ceramics Research Center, Nagoya Institute of Technology, Gokiso-cho, Showa-ku, Nagoya, Aichi 466-8555, Japan. E-mail: shirai@nitech.ac.jp milling of bulk $\mathrm{Si}^{\mathbf{1 1}}$ have been demonstrated. As for bottom-up approaches, Si NCs have been synthesized via the plasma decomposition of silane, ${ }^{12}$ chemical reduction of tetrachloride silane, ${ }^{\mathbf{1 3}}$ thermal pyrolysis of hydrogen silsesquioxane, ${ }^{\mathbf{1 4}}$ and thermal disproportionation of $\mathrm{SiO}_{x}(1<x<2) .{ }^{15}$

$\mathrm{SiO}$, an amorphous monoxide material, has been widely utilized in electronic devices, such as in anode materials for lithium ion batteries, insulating layers in integrated circuits, etc. ${ }^{16}$ And as a commercially available material, SiO is also utilized as a promising precursor for Si NC synthesis through thermal disproportionation reactions. Rybaltovskiy et al. previously reported the synthesis of $\mathrm{Si} / \mathrm{SiO}_{x}$ core/shell nanoparticles by thermal disproportionation of $\mathrm{SiO} .{ }^{17}$ Sun et al. reported the synthesis of free-standing Si NCs on a large-scale, based on thermal disproportionation of SiO. ${ }^{18}$ However, both of these synthesized $\mathrm{Si} / \mathrm{SiO}_{x}$ core/shell nanoparticles and $\mathrm{Si}$ NCs perform photoluminescence in the infrared wavelength region. Also, high temperatures of more than $900{ }^{\circ} \mathrm{C}$ are required for the synthesis of Si NCs via the thermal disproportionation reaction of SiO. Furthermore, the size of Si NCs must be tuned through critical temperature increasing. ${ }^{17-20} \mathrm{In}$ this work, we reported a novel and facile route for the synthesis of visible photoluminescence Si NCs via mechanochemical disproportionation of SiO under room temperature. The size of synthesized Si NCs can be easily tuned in the 2$5 \mathrm{~nm}$ order by the mechanochemical conditions. After efficient surface passivation, our synthesized Si NCs exhibit visible photoluminescence with a maximum emission peak around $450 \mathrm{~nm}$. Excitation wavelength-dependent photoluminescence behaviour is confirmed, which suggests that the photoluminescence mechanism is attributed to the size dependent wide bandgap in synthesized Si NCs based on quantum confinement effects. To the best of our knowledge, this is the first report of the synthesis of visible photoluminescence $\mathrm{Si}$ NCs via a low-temperature disproportionation reaction system. In addition, the reaction mechanism of mechanochemical disproportionation was also investigated. 
Si NCs were prepared by mechanochemical ball-milling of $3 \mathrm{~g}$ SiO (Osaka Titanium Technology, Co. Ltd) with $40 \mathrm{~g} \mathrm{ZrO}_{2}$ ball under an argon atmosphere. The size of the $\mathrm{ZrO}_{2}$ ball utilized in this study is $15 \mathrm{~mm}$ and the milling duration was settled as 1, 3 and 5 hours, respectively. Free-standing Si NCs were liberated via chemical etching of $50 \mathrm{mg}$ mechanochemical disproportionation product by a mixture of hydrofluoric acid (HF, 49\%, $3 \mathrm{ml}) /$ hydrochloric acid $(37 \%, 75 \mu \mathrm{l}) /$ ethanol $(0.75$ $\mathrm{ml}$ ). The reaction was conducted in the dark (to maintain the size of Si NCs) for 5 hours in a PTFE container. The Si NCs after chemical etching were extracted by $30 \mathrm{ml}$ toluene solvent twice and collected via centrifugation at $9000 \mathrm{rpm}$ for 20 minutes. The surface of the Si NCs was passivated by 1-decene after chemical etching by HF solution to increase the stability and optical properties of the Si NCs. $10 \mathrm{ml}$ 1-decene was added into etched Si NCs and the solution was heated up to $165^{\circ} \mathrm{C}$ under an argon atmosphere. After reacting for 13 hours, the solution was mixed with $20 \mathrm{ml}$ methanol/ethanol $(1: 3)$ solution to remove the excess 1-decene via high-speed centrifugation $(20000 \mathrm{~g}, 20$ minutes). Si NCs were collected and purified via an anti-solvent/ solvent system by using methanol/toluene with volume ratio of $3: 1$ for three cycles, assisted via high-speed centrifugation (20 000g, 20 minutes for each cycle). Finally, pure and clean toluene was added to the Si NCs, whose solution was filtered through a PTFE membrane with pore size of $200 \mathrm{~nm}$, and then the Si NC solution was ready for further characterizations. The synthesis procedure in this study is summarized in Scheme 1.

The crystal structures of $\mathrm{SiO}$ raw powder and mechanochemically treated powders were analyzed by X-ray diffraction (XRD) measurement (Rigaku). The XRD measurement is carried out by $\mathrm{Cu} \mathrm{K} \alpha$ radiation with an acceleration voltage and current of $40 \mathrm{kV}$ and $40 \mathrm{~mA}$. The chemical structure was also characterized by Raman spectroscopy (Renishaw) with a $532 \mathrm{~nm}$ laser. The chemical states of the SiO raw powder and mechanochemically treated powder were investigated by X-ray photoelectron spectrometry (XPS; M-probe SSX-100, S.S.I, USA) with Al $\mathrm{K} \alpha \mathrm{X}$-ray $(h \nu=1486.6 \mathrm{eV})$ radiation. The shift of the binding energy due to relative surface charge was corrected using the Au4f level at $83.98 \mathrm{eV}$. The size and morphology of the Si NCs were confirmed by transmission electron microscopy (TEM, JEOL, JEM-2100F). The photoluminescence spectrum of surface-passivated Si NCs as a toluene dispersion was recorded on a fluorescence spectrometer (JASCO), and the surface and size were confirmed by Fourier transform infrared spectroscopy (FTIR; JASCO) and a dynamic light scattering (DLS; Zetasizer, Malvern) system.

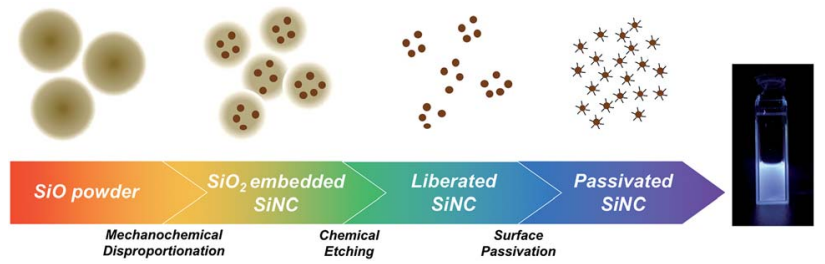

Scheme 1 Synthesis procedure of Si NCs (photograph: emissive Si NCs).
Fig. 1 shows the XRD patterns of raw and mechanochemically treated SiO powders. As for the SiO raw powder, only a halo peak at $21^{\circ}$ according to the amorphous component is observed. In the case of mechanochemically treated SiO powder, three sharp peaks appear at $28^{\circ}, 47^{\circ}$ and $56^{\circ}$, corresponding to (111), (220) and (311) planes of crystalline Si..$^{2,14-20}$ According to the Scherrer formula, the size of the crystalline $\mathrm{Si}$ domain is increased as the mechanochemical treatment proceeds. We suggest that the crystalline $\mathrm{Si}$ is generated through the disproportionation of SiO. Fig. 1b illustrates the Raman spectra of raw and mechanochemically treated $\mathrm{SiO}$ powders. For raw SiO, a wide peak at $475 \mathrm{~cm}^{-1}$ corresponding to amorphous $\mathrm{Si}$ is observed, whose peak intensity degraded for SiO after mechanochemical treatment. Additionally, a crystalline Si peak appeared in mechanochemically treated $\mathrm{SiO}$ and its Raman shift is red-shifted through treatment processing, which demonstrates that the size of the crystalline Si domain increases. ${ }^{17}$ Such results from Raman spectra show good agreement with XRD results.

In order to confirm the morphology of the crystalline Si domain in the product, TEM measurement was conducted on the samples. As the images in Fig. 2 show, the raw powder of SiO shows amorphous phase, while Si NCs appear in the amorphous phase after mechanochemical treatment. The lattice fringe with a $d$-spacing of $1.9 \AA$ is observed, which can be assigned as the (220) plane of crystalline Si. ${ }^{14-20}$ The sizes of Si NCs observed for 1,3 and 5 hours mechanochemical treatment are about 2, 3 and $5 \mathrm{~nm}$. These sizes of Si NCs increased as the mechanochemical treatment time increased, and these results show good agreement with the XRD results given in Fig. 1.
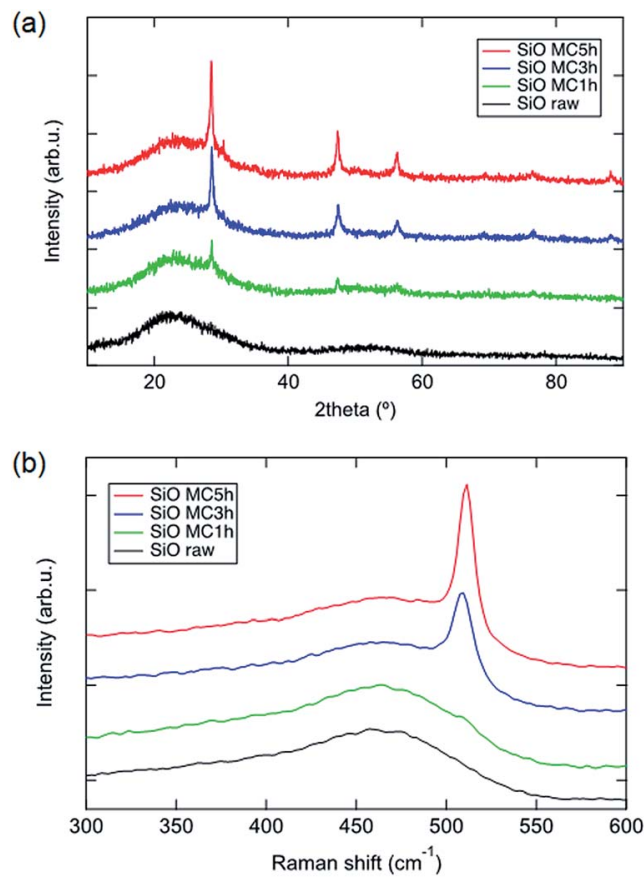

Fig. 1 The XRD patterns (a) and Raman spectra (b) of raw and mechanochemically treated $\mathrm{SiO}$. 

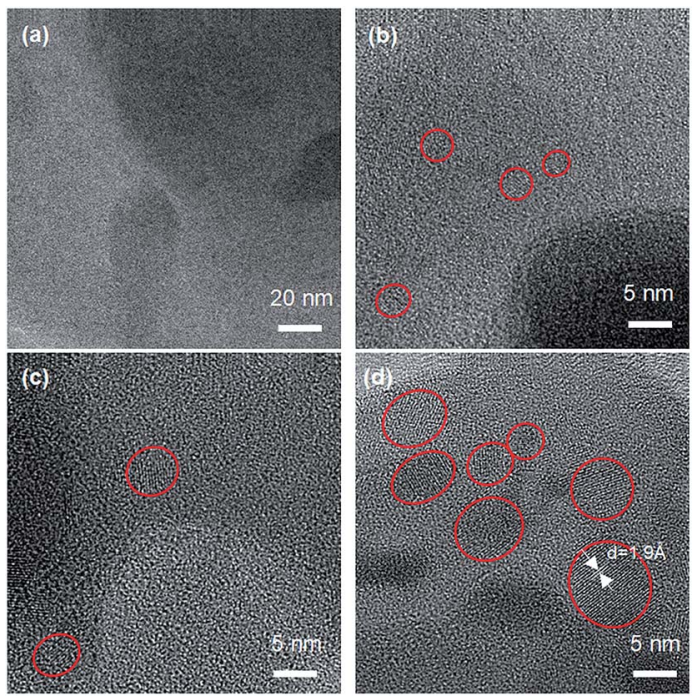

Fig. 2 TEM images of raw (a) and mechanochemically treated SiO for different durations of (b) 1 hour, (c) 3 hours and (d) 5 hours.

To investigate the reaction mechanism of this room temperature mechanochemical disproportionation, the chemical state of $\mathrm{Si}$ was analysed by XPS measurement. Fig. 3 demonstrates the XPS spectra of the Si 2p orbital for raw and mechanochemically treated SiO. The spectra are fitted as five peaks at 99.7, 100.6, 101.6, 102.7 and $103.8 \mathrm{eV}$ by Gaussian equations, corresponding to $\mathrm{Si}^{0}, \mathrm{Si}^{+}, \mathrm{Si}^{2+}, \mathrm{Si}^{3+}$ and $\mathrm{Si}^{4+}$ states, respectively. ${ }^{21,22}$ The calculated peak area of each component is summarized as the insets of Fig. 3, which illustrate that the amount of $\mathrm{Si}^{0}$ and $\mathrm{Si}^{4+}$ states increased with a decrease of low covalent states like $\mathrm{Si}^{+}$and $\mathrm{Si}^{2+}$ and $\mathrm{Si}^{3+}$ after mechanochemical

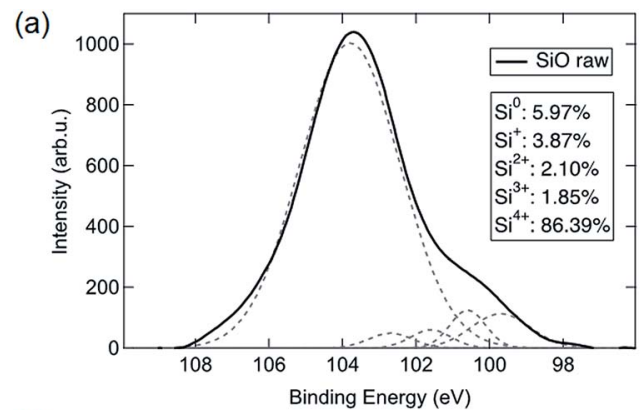

(b)

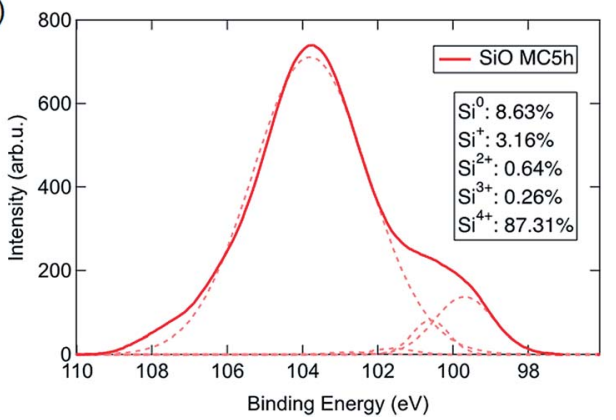

Fig. 3 XPS spectra of the Si $2 p$ orbital of raw and mechanochemically treated $\mathrm{SiO}$ treatment. These results suggest that a disproportionation reaction on SiO occurs during room temperature mechanochemical treatment and Si NCs in a $\mathrm{SiO}_{2}$ matrix are generated.

Regarding to XRD, Raman, TEM and XPS results, here we explain the mechanism for the formation of Si NCs as the following three steps. Firstly, Si radicals and Si oxide radicals are generated by the application of mechanical stress during the high-energy planetary ball-milling, whose process should be similar to mechanochemical processing of quartz compounds. ${ }^{23}$ The generation of radicals has been demonstrated as a main factor for mechanochemical reaction of mixed solid phases..$^{24,25}$ In addition, the generated Si radicals are stable in the inert atmosphere of argon gas. Secondly, the generated small amount of Si radicals may diffuse through the concentrated SiO matrix based on the Gorsky effect. Such a diffusion mechanism is widely utilized in the mechanochemical synthesis of alloy compounds. Thirdly, Si NC domains are formed by the gathering of Si radicals. ${ }^{24,25}$ The above-described three-step reaction occurs continuously during ball-milling and it can also well explain the growth of the NC size by increasing the milling time.

Next, free-standing Si NCs are liberated via HF chemical etching of $\mathrm{SiO}_{2}$, and then the surface of the $\mathrm{NC}$ is passivated by hydrocarbon solvent to ensure the stability and solubility in organic solvent. ${ }^{26,27}$ Fig. 4 displays the photoluminescence spectra of Si NCs observed from 5 hours mechanochemically treated SiO. Visible emission with wavelength ranges from $400 \mathrm{~nm}$ to $600 \mathrm{~nm}$ is observed with a strong emission peak at $450 \mathrm{~nm}$. The emission peak is red-shifted as the excitation wavelength red-shifts, and this phenomenon is attributed to the size-tuned bandgaps of the observed Si NCs based on the quantum confinement effect. ${ }^{1}$ As the photograph of emissive $\mathrm{Si}$ NCs in Scheme 1 shows, a bright blue colour is observed when excited by a UV light source. To confirm the photoluminescence mechanism, the surface state of the passivated Si NCs is measured by ATR-FTIR. According to the ATR-FTIR spectra shown in Fig. 5a, the peaks of $\mathrm{C}-\mathrm{H}$ stretching around $2900 \mathrm{~cm}^{-1}$, C-H scissoring and methyl rock around $1470 \mathrm{~cm}^{-1}$, long-chain methyl rock at $723 \mathrm{~cm}^{-1}$ without any existence of SiO-Si stretching at $1100 \mathrm{~cm}^{-1}$ and Si-H stretching at $2100 \mathrm{~cm}^{-1}$ demonstrate that the surface of Si NCs is completely passivated with 1-decene without any oxidation. By referring to several

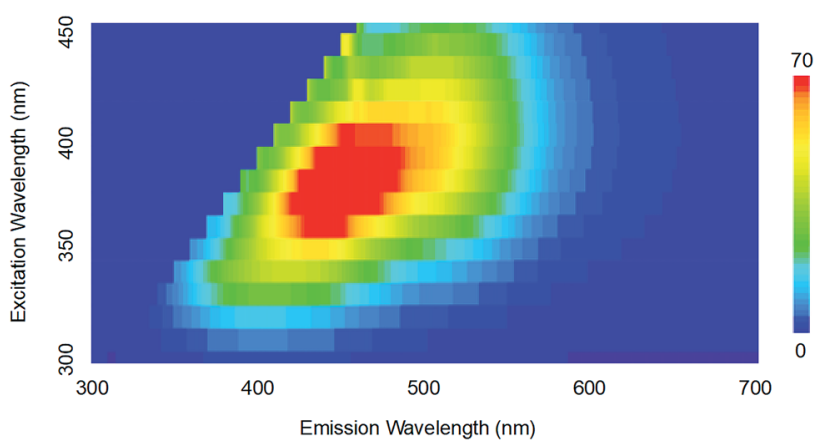

Fig. 4 The 3D photoluminescence spectra of Si NCs under different excitation wavelengths (color bar: photoluminescence intensity). 

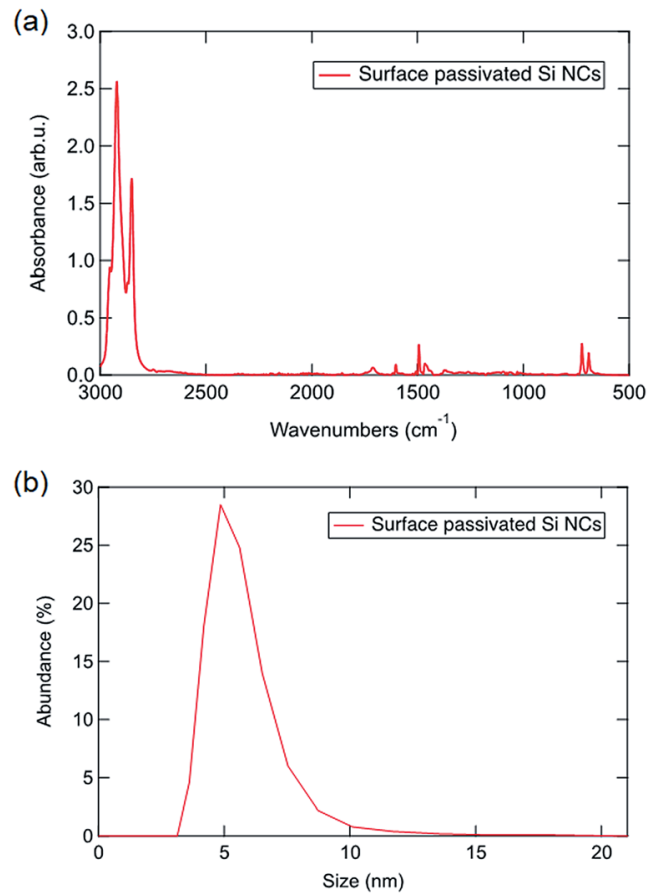

Fig. 5 FTIR spectra and DLS size distribution for surface passivated Si NCs.

experimental and theoretical studies of photoluminescence mechanisms for alkyl-passivated Si $\mathrm{NCs},{ }^{28,29}$ we conclude that the excitation-wavelength-dependent photoluminescence of our Si NCs must be attributed to the electron and hole recombination of the size-dependent direct-like bandgap of core state due to quantum confinement effects, where the wavefunctions of electron and hole are modified through surface passivation by alkyl groups. Additionally, the size distribution of surface passivated Si NCs in solution is evaluated by DLS. As is shown in Fig. 5b, the hydrodynamic size of Si NCs is $5.45 \pm 1.39 \mathrm{~nm}$, and this value shows good agreement with the TEM result.

\section{Conclusions}

In conclusion, visible photoluminescence Si NCs are successfully synthesized via a novel and facile room temperature mechanochemical disproportionation of SiO. The size of Si NCs can be tuned by the mechanochemical treatment time, where the Si NC size increases as the treatment time increases based on the results of XRD and TEM characterizations. Based on the XPS analysis and calculating the component of each chemical state of $\mathrm{Si}$, the disproportionation reaction is confirmed. The synthesized Si NCs exhibit bright blue emission with an excitation wavelength dependent photoluminescence in the visible region. Compared with the conventional thermal disproportionation method, the synthesis process through a room temperature mechanochemical process and easy size-tuning in this paper opens a new strategy for the synthesis of Si NCs. In addition, the Si NCs synthesized in the current work perform visible photoluminescence through the electron and hole recombination between the quantum confined wide bandgap of the core state, while the Si NCs obtained from thermal disproportionation only show photoluminescence in the infrared wavelength region. This strategy is also expected to be available for the synthesis of other functional NCs like Ge NCs and Sn NCs through such room temperature mechanochemical disproportionation of GeO and SnO.

\section{Conflicts of interest}

There are no conflicts to declare.

\section{Notes and references}

1 K. Dohnalova, T. Gregorkiewics and K. Kusova, J. Phys.: Condens. Matter, 2014, 26, 173201.

2 J. G. C. Veinot, Chem. Commun., 2016, 4160.

3 F. Maier-Flaig, J. Rinck, M. Stephan, T. Bocksrocker, M. Bruns, C. Kubel, A. K. Powell, G. A. Ozin and U. Lemmer, Nano Lett., 2013, 13, 475.

4 Y. Xin, K. Kitasako and K. Saitow, Appl. Phys. Lett., 2015, 106, 201102.

5 H. Tan, Z. Ni, W. Peng, S. Du, X. Liu, S. Zhao, W. Li, Z. Ye, M. Xu, Y. Xu, X. Pi and D. Yang, Nano Energy, 2018, 52, 422.

6 J. M. Kim, S. Kim, D. H. Shin, S. W. Seo, H. S. Lee, J. H. Kim, C. W. Jang, S. S. Kang, S.-H. Choi, G. Y. Kwak, K. J. Kim, H. Lee and H. Lee, Nano Energy, 2018, 43, 124.

7 F. Meinardi, S. Ehrenberg, L. Dhamo, F. Carulli, M. Mauri, F. Bruni, R. Simonutti, U. Kortshagen and S. Brovelli, Nat. Photonics, 2017, 11, 177.

8 Z. Ni, L. Ma, S. Du, Y. Xu, M. Yuan, H. Fang, Z. Wang, M. Xu, D. Li, J. Yang, W. Hu, X. Pi and D. Yang, ACS Nano, 2017, 11, 9854.

9 Y. Xin, T. Kitasako, M. Maeda and K. Saitow, Chem. Phys. Lett., 2017, 674, 90.

10 Z. Kang, Y. Liu, C. H. A. Tsang, D. Duo Duo Ma, X. Fan, N.-B. Wong and S.-T. Lee, Adv. Mater., 2009, 21, 661.

11 A. S. Heintz, M. J. Fink and B. Mitchell, Adv. Mater., 2007, 19, 3984.

12 L. Mangolini, E. Thimsen and U. Kortshagen, Nano Lett., 2005, 5, 655.

13 K. Dohnalova, A. Fucikova, C. P. Umesh, J. Humpolickova, J. M. Paulusse, J. Valenta, H. Zuilhof, M. Hof and T. Gregorkiewicz, Small, 2012, 8, 3185.

14 Y. Xin, R. Wakimoto and K. Saitow, Chem. Lett., 2017, 46, 699.

15 F. Iacona, C. Bongiorno and C. Spinella, J. Appl. Phys., 2004, 95, 3723.

16 A. Hirata, S. Kohara, T. Asada, M. Arao, C. Yogi, H. Imai, Y. Tan, T. Fujita and M. Chen, Nat. Commun., 2015, 7, 11591. 17 A. O. Rybaltovskiy, A. A. Ischenko, Y. S. Zavorotny, A. V. Garshev, S. G. Dorofeev, N. N. Kononov, N. V. Minaev, S. A. Minaeva, A. P. Sviridoc, P. S. Timashev, I. I. Khodos, V. I. Yusupov, M. A. Lazov, V. Y. a. Panchenoko and V. N. Bagratashvili, J. Mater. Sci., 2015, 50, 2247.

18 W. Sun, C. Qian, X. S. Cui, L. Wang, M. Wei, G. Casillas, A. S. Helmy and G. A. Ozin, Nanoscale, 2016, 8, 3678. 
19 W. Sun, C. Qian, L. Wang, M. Wei, M. L. Mastronardi, G. Casilla, J. Breu and G. A. Ozin, Adv. Mater., 2015, 27, 746. 20 S. G. Dorofeev, A. A. Ishchenko, N. N. Kononov and G. V. Fetisov, Curr. Appl. Phys., 2012, 12, 718.

21 C. H. Hessel, D. Reid, M. G. Panthani, M. R. Rasch, B. W. Goodfellow, J. Wei, H. Fujii, V. Akhavan and B. A. Korgel, Chem. Mater., 2012, 24, 393.

22 C. M. Hessel, E. J. Henderson and J. G. C. Veinot, J. Phys. Chem. C, 2007, 111, 6956.

23 U. Voland, R. Schrader and H. Schneider, Z. Anorg. Allg. Chem., 1969, 368, 317.
24 E. Boldyreva, Chem. Soc. Rev., 2013, 42, 7719.

25 V. V. Zyryanov, Sci. Sintering, 2005, 37, 77.

26 J. R. R. Nunez, J. A. Kelly, E. J. Henderson and J. G. Veinot, Chem. Mater., 2012, 24, 346.

27 D. P. Puzzo, E. J. Henderson, M. G. Helander, Z. Wang, G. A. Ozin and Z. Lu, Nano Lett., 2011, 11, 1585.

28 K. Dohnalova, A. N. Poddubny, A. A. Prokofiev, W. DAM de Boer, C. P. Umesh, J. MJ Paulusse, H. Zuilhof and T. Gregorkiewicz, Light: Sci. Appl., 2013, 2, e47.

29 F. A. Reboredo and G. Galli, J. Phys. Chem. B, 2005, 109, 1072. 